\title{
PERTURBATION SOLUTIONS FOR MAGNETOHYDRODYNAMICS (MHD) FLOW OF IN A NON-NEWTONIAN FLUID BETWEEN CONCENTRIC CYLINDERS
}

\author{
M. YÜRÜSOY* and Ö.F. GÜLER \\ Department of Mechanical Engineering, Technology Faculty \\ Afyon Kocatepe University \\ 03200 Afyonkarahisar, TURKEY \\ E-mail: yurusoy@aku.edu.tr
}

\begin{abstract}
The steady-state magnetohydrodynamics (MHD) flow of a third-grade fluid with a variable viscosity parameter between concentric cylinders (annular pipe) with heat transfer is examined. The temperature of annular pipes is assumed to be higher than the temperature of the fluid. Three types of viscosity models were used, i.e., the constant viscosity model, space dependent viscosity model and the Reynolds viscosity model which is dependent on temperature in an exponential manner. Approximate analytical solutions are presented by using the perturbation technique. The variation of velocity and temperature profile in the fluid is analytically calculated. In addition, equations of motion are solved numerically. The numerical solutions obtained are compared with analytical solutions. Thus, the validity intervals of the analytical solutions are determined.
\end{abstract}

Key words: MHD flow, perturbation technique, annular flow, third grade fluid.

\section{Introduction}

There are many applications of non-Newtonian fluids in the industry. Therefore, many theoretical and experimental studies have been carried out on non-Newtonian fluid models. The most widely used model is undoubtedly the third-grade fluid model. Studies on the third order fluid model are more concerned with the boundary layer flow and the flows in the pipe. There are many applications of the studies on the boundary layer flow and the pipe flow. The most important applications of the boundary layer theory are food processing, the extrusion of polymers or the production of plastic films, lubrication, continuous casting etc. The most common applications of the flows between concentric cylinders (annular pipe) with heat transfer are heat exchangers. Manufacturing rate and temperature changes are the most important factors in heat exchangers. For this purpose, a number of analytical studies have been carried out for non-Newtonian fluid flow in the pipe, also for boundary layer flows. In particular, studies for boundary layer flow using nonNewtonian model are shown.

Yürüsoy and Pakdemirli [1] solved the boundary layer equation of a laminar flow for third grade fluids. Multi-layer boundary layer equations have been studied for second and third grade fluids by Pakdemirli [2]. Hayat and Kara [3] analyzed parallel plates flow of a third-order fluid by using the Lie Groups theory. Hayat et al. [4] found analytical solutions for the flow of a third-order fluid in a porous half space by using the Homotopy Analysis Method (HAM). Pakdemirli et al. [5] produced analytical solutions for a modified second grade fluid by using the perturbation method. Pakdemirli et al. [6] solved laminar and steady state boundary layer equations of power-law fluids of second grade by using similarity transformation and numerical technique. By using similarity transformation, Chamkha [7] studied the thermal boundary layer on a stretching sheet for a power-law fluid. The effect of suction or injection on the laminar boundary

\footnotetext{
* To whom correspondence should be addressed
} 
layer approach over a stretching sheet or moving surface for third grade fluids was analyzed by Ali and Yurusoy [8].

Studies on the non-Newtonian fluid flow in the pipe are as follows. The work of Massoudi and Christie [9], which deals with the third-order fluid flow for heat transfer and variable viscosity state. The study also considers as a function of temperature and a numerical method was used for the solution of this problem. A study of approximate analytical solutions was carried out by Yürüsoy and Pakdemirli [10]. Approximate analytical solutions [10], by using perturbation technique, are compared with the numerical solution given by Massoudi and Christie [9]. This work has also pioneered analytical solutions for both pipe flow and flow between parallel plates. Many similar works have been carried out [11-13]. In all these studies, analytical solutions were generally made using perturbation techniques and Vogel and Reynolds viscosity models were used as was given in [10]. Viscosity models are considered as a function of temperature.

In this study, the steady-state magnetohydrodynamics (MHD) flow of a viscous third-grade fluid between concentric cylinders (annular pipe) with heat transfer is considered. Three types of viscosity models were used. These are the constant viscosity model, space dependent viscosity model, and the Reynolds viscosity model which depends on temperature in an exponential manner. The equations of motion, highly non-linear ordinary differential equations systems, are solved using the regular perturbation technique for three types of viscosity models. The new analytical solutions for an annular pipe flow are compared with numerical results for all physical parameters (non-Newtonian, viscosity and heat generation parameters, etc.) and an excellent agreement is observed.

\section{Equations of motion}

The schematic of an MHD (magnetohydrodynamics) flow of a third grade fluid through an annular pipe is shown in Fig.1. The viscosity of the fluid is taken as variable. The pressure gradient in the annular pipe is considered as constant. The continuity, momentum and energy equations are

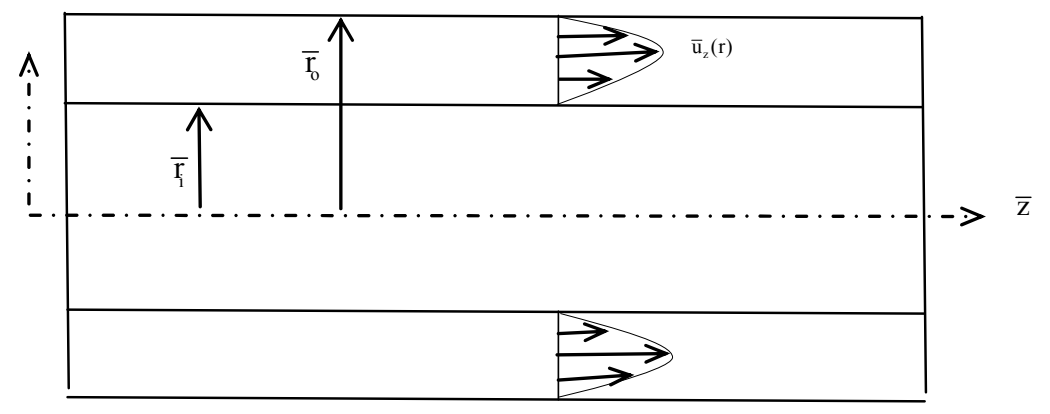

Fig.1. Schematic view of the annular pipe and flow situation.

$$
\begin{aligned}
& \operatorname{div}(\boldsymbol{V})=0, \\
& \rho \frac{d \boldsymbol{V}}{d t}=\operatorname{div}(\boldsymbol{T})+\boldsymbol{J} \times \boldsymbol{B}, \\
& \rho c_{p} \frac{d \theta}{d t}=\boldsymbol{T} \times \boldsymbol{L}+k \nabla^{2} \theta
\end{aligned}
$$

where $c_{p}$ is the specific heat, $\theta$ is the non-Newtonian fluid temperature, $\boldsymbol{J}$ is the density of the electric current, $\boldsymbol{B}$ is the magnetic field, $\boldsymbol{L}$ is the velocity gradient. The stress constitutive equation for third grade fluids is defined by 


$$
\boldsymbol{T}=-p \boldsymbol{I}+\mu \boldsymbol{A}_{1}+\alpha_{1} \boldsymbol{A}_{2}+\alpha_{2} \boldsymbol{A}_{\boldsymbol{I}}^{2}+\beta\left(\operatorname{tr} \boldsymbol{A}_{1}^{2}\right) \boldsymbol{A}_{\boldsymbol{I}}
$$

where $\boldsymbol{T}$ is the stress tensor, $p$ is the pressure, $\mu$ is the viscosity, $\alpha_{1}, \alpha_{2}, \beta$ are material constants, $\boldsymbol{A}_{1}$ and $\boldsymbol{A}_{2}$.are Rivlin-Ericksen tensors.

The velocity field and temperature are as follows

$$
\boldsymbol{V}=\left[0,0, u_{z}(r)\right], \quad \theta(r) .
$$

When Eq.(2.5) is taken into account, Eqs (2.1)-(2.3) are reduced to the form below.

$$
\begin{aligned}
& \frac{1}{\bar{r}} \frac{d}{d r}\left(\bar{\mu} \bar{r} \frac{d \bar{u}_{z}}{d \bar{r}}\right)+\frac{2 \beta}{\bar{r}} \frac{r}{d \bar{r}}\left(r\left(\frac{d \bar{u}_{z}}{d \bar{r}}\right)^{3}\right)=\bar{C}+\sigma B_{0}^{2} \bar{u}_{z} \\
& \bar{\mu}\left(\frac{d \bar{u}_{z}}{d \bar{r}}\right)^{2}+2 \beta\left(\frac{d \bar{u}_{z}}{d \bar{r}}\right)^{4}+k\left(\frac{1}{\bar{r}} \frac{d}{d \bar{r}}\left(\bar{r} \frac{d \bar{\theta}}{d \bar{r}}\right)\right)=0
\end{aligned}
$$

Boundary conditions of the problem are

$$
\begin{aligned}
& \bar{u}_{z}\left(\bar{r}_{i}\right)=0, \bar{u}_{z}\left(\bar{r}_{o}\right)=0, \\
& \theta\left(\bar{r}_{i}\right)=0, \quad \theta\left(\bar{r}_{o}\right)=0
\end{aligned}
$$

where $\sigma$ is the electrical conductivity of the third grade fluid, $\bar{r}$ is the dimensional radius $\left(\overline{r_{i}}<\bar{r}<\bar{r}_{o}\right), \bar{r}_{i}$ is the dimensional radius of the inner cylinder, $\bar{r}_{o}$ is the dimensional radius of the outer cylinder, $\bar{u}_{z}$ is the dimensional velocity, $\bar{\theta}$ is the dimensional temperature, $\bar{\mu}$ is the dimensional viscosity.

The dimensionless parameters for the problem are defined as follows

$$
\begin{aligned}
& r=\frac{\bar{r}}{\bar{r}_{i}}, \quad R=\frac{\bar{r}_{o}}{\bar{r}_{i}}, \quad u_{z}=\frac{\bar{u}_{z}}{V_{0}}, \quad \mu=\frac{\bar{\mu}}{\mu_{0}}, \quad \frac{\bar{\theta}-\theta_{0}}{\theta_{1}-\theta_{0}}, \quad \Lambda=\frac{2 \beta V_{0}^{2}}{\mu_{0} \bar{r}_{i}^{2}}, \\
& \Gamma=\frac{\mu_{0} V_{0}}{k\left(\theta_{1}-\theta_{0}\right)}, \quad C=\frac{\bar{C} \bar{r}_{i}^{2}}{\mu_{0} V_{0}}, \quad M=\frac{\sigma B_{0}^{2} \bar{r}_{i}^{2}}{\mu_{0}}
\end{aligned}
$$

where $V_{0}$ is the reference velocity, $\mu_{0}$ is the reference viscosity, $\theta_{0}$ is the reference temperature, $\theta_{l}$ is the fluid temperature, $C$ is the pressure drop in the axial direction, $k$ is the thermal conductivity, $\Lambda$ is the dimensionless non-Newtonian parameter, $\Gamma$ is the Brinkman number, $B_{0}$ is the magnetic field applied to the fluid, $M$ is the magnetic parameter.

Equations (2.6)-(2.8) are non-dimensionalized as

$$
\begin{aligned}
& \frac{d \mu}{d r} \frac{d u_{z}}{d r}+\mu \frac{d^{2} u_{z}}{d r^{2}}+\mu \frac{1}{r} \frac{d u_{z}}{d r}+\frac{\Lambda}{r}\left(\frac{d u_{z}}{d r}\right)^{3}+3 \Lambda\left(\frac{d u_{z}}{d r}\right)^{2} \frac{d^{2} u_{z}}{d r^{2}}=C+M u_{z}, \\
& \frac{d^{2} \theta}{d r^{2}}+\frac{1}{r} \frac{d \theta}{d r}+\Gamma \mu\left(\frac{d u_{z}}{d r}\right)^{2}+\Gamma \Lambda\left(\frac{d u_{z}}{d r}\right)^{4}=0,
\end{aligned}
$$




$$
u_{z}(l)=0, u_{z}(R)=0, \quad \theta(l)=0, \quad \theta(R)=0
$$

Solutions will be presented for different viscosity models.

\section{Perturbation solutions}

In this section, solutions for two different viscosity models will be presented. The equations of motion, highly non-linear ordinary equations systems will be solved using the regular perturbation technique.

Case 1: Reynolds Model

The Reynolds viscosity model depends on temperature in an exponential type [1].

$$
\mu=e^{-\alpha \theta}
$$

When $\alpha=0$, the model corresponds to constant viscosity. One assumes expansion for velocity and temperature as follows

$$
\begin{aligned}
& u_{z}=u_{z}^{0}+\varepsilon u_{z}^{1}+O\left(\varepsilon^{2}\right), \\
& \theta=\theta_{0}+\varepsilon \theta_{1}+O\left(\varepsilon^{2}\right) .
\end{aligned}
$$

Here, we shall select

$$
\Lambda=\varepsilon \lambda, \quad \alpha=\varepsilon \bar{\alpha}, \quad M=\varepsilon m .
$$

Viscosity function and its derivative form now read approximately as follows

$$
\mu=1-\varepsilon m \theta, \quad \frac{d \mu}{d r}=-\varepsilon \theta^{\prime}
$$

Substituting all into the original equations of motion and separating at each order of $\varepsilon$, one has:

For $O(1)$ :

$$
\begin{aligned}
& r\left(u_{z}^{0}\right)^{\prime \prime}+\left(u_{z}^{0}\right)^{\prime}=C r, \\
& \theta_{0}^{\prime \prime}+\frac{1}{r} \theta_{0}^{\prime}=-\Gamma\left(\left(u_{z}^{0}\right)^{\prime}\right)^{2}, \\
& u_{z}^{0}(1)=0, \quad \theta(1)=0 .
\end{aligned}
$$

For $O(\varepsilon)$ : 


$$
\begin{aligned}
& r\left(u_{z}^{l}\right)^{\prime \prime}+\left(u_{z}^{l}\right)^{\prime}=\hat{\alpha} \theta_{0}\left(u_{z}^{0}\right)^{\prime}+\hat{\alpha} r \theta_{0}\left(u_{z}^{0}\right)^{\prime \prime}+\hat{\alpha} r \theta_{0}^{\prime}\left(u_{z}^{0}\right)^{\prime}-\lambda\left(\left(u_{z}^{0}\right)^{\prime}\right)^{3}-3 \lambda r\left(\left(u_{z}^{0}\right)^{\prime}\right)^{2}\left(u_{z}^{0}\right)^{\prime \prime}+m r u_{z}^{0}, \\
& \theta_{l}^{\prime \prime}+\frac{1}{r} \theta_{l}^{\prime}=-\Gamma\left(\left(u_{z}^{0}\right)^{\prime}\right)^{2}\left(-\hat{\alpha} \theta_{0}+\lambda\left(\left(u_{z}^{0}\right)^{\prime}\right)^{2}\right)-2 \Gamma\left(u_{z}^{0}\right)^{\prime}\left(u_{z}^{l}\right)^{\prime}, \\
& u_{z}^{I}(R)=0, \theta_{l}(R)=0 .
\end{aligned}
$$

For the first order, solutions are

$$
\begin{aligned}
& u_{z}^{0}=t_{1}\left(r^{2}-1\right)+t_{2} \ln (r), \\
& \theta_{0}=t_{3} r^{4}+t_{4} r^{2}+t_{5}(\ln (r))^{2}+d_{1} \ln (r)+d_{2} .
\end{aligned}
$$

Substitute these solutions into Eqs (3.6) and by integrating the resulting equations twice, one obtains

$$
\begin{aligned}
& u_{z}^{1}=t_{6} r^{6}+t_{7} r^{4}+t_{8} r^{2}+t_{9} r^{2}(\ln (r))^{2}+t_{10} r^{2} \ln (r)+t_{11}(\ln (r))^{3}+t_{12}(\ln (r))^{2}+\frac{t_{13}}{r^{2}}+e_{1} \ln (r)+e_{2}, \\
& \theta_{1}=t_{14} r^{8}+t_{15} r^{6}+t_{16} r^{4}+t_{17} r^{4}(\ln (r))^{2}+t_{18} r^{4} \ln (r)+t_{19} r^{2}+t_{20} r^{2}(\ln (r))^{2}+t_{21} r^{2} \ln (r)+ \\
& +t_{22}(\ln (r))^{4}+t_{23}(\ln (r))^{3}+t_{24}(\ln (r))^{2}+\frac{t_{25}}{r^{2}}+f_{1} \ln (r)+f_{2}
\end{aligned}
$$

where $t_{1,2,3 \ldots 25}, d_{1}, d_{2}, e_{1}, e_{2}, f_{l}$ and $f_{2}$ are

$$
\begin{aligned}
& t_{1}=\frac{C}{4}, \quad t_{2}=-\frac{C\left(R^{2}-1\right)}{4 \ln (R)}, \quad t_{3}=-\frac{\Gamma t_{1}^{2}}{4}, \quad t_{4}=-\Gamma t_{1} t_{2}, \\
& t_{5}=-\frac{\Gamma t_{2}^{2}}{2}, \quad t_{6}=\frac{\hat{\alpha} t_{1} t_{3}}{3}, \quad t_{7}=\frac{\hat{\alpha} t_{1} t_{4}}{2}+\frac{\hat{\alpha} t_{3} t_{2}}{4}-2 t_{1}^{3} \lambda+\frac{m t_{1}}{16}, \\
& t_{8}=-\frac{\hat{\alpha} d_{1} t_{1}}{2}+\hat{\alpha} d_{2} t_{1}+\frac{\hat{\alpha} t_{2} t_{4}}{2}-6 \lambda t_{2} t_{1}^{2}-\frac{m t_{2}}{4}-\frac{m t_{1}}{4}+\frac{\hat{\alpha} t_{1} t_{5}}{2}, \\
& t_{9}=\hat{\alpha} t_{1} t_{5}, \quad t_{10}=-\hat{\alpha} t_{5} t_{1}+\hat{\alpha} d_{1} t_{1}+\frac{m t_{2}}{4},
\end{aligned}
$$




$$
\begin{aligned}
& t_{11}=\frac{\hat{\alpha} t_{2} t_{5}}{3}, \quad t_{12}=\frac{\hat{\alpha} d_{1} t_{2}}{2}, \quad t_{13}=\frac{\lambda t_{2}^{3}}{2}, \quad t_{14}=-\frac{3 \Gamma t_{1} t_{6}}{8}+\frac{\Gamma \hat{\alpha} t_{1}^{2} t_{3}}{16}, \\
& t_{15}=-\frac{4 \Gamma \lambda t_{1}^{4}}{9}-\frac{\Gamma t_{2} t_{6}}{3}-\frac{4 \Gamma t_{1} t_{7}}{9}+\frac{\Gamma \hat{\alpha} t_{1} t_{7}}{9}+\frac{\Gamma \hat{\alpha} t_{1} t_{2} t_{3}}{9}, \\
& t_{16}=-\frac{\Gamma t_{2} t_{7}}{2}-\frac{\Gamma t_{1} t_{8}}{2}+\frac{\Gamma t_{1} t_{9}}{16}+\frac{3 \Gamma \hat{\alpha} t_{1}^{2} t_{5}}{32}-\frac{\Gamma \hat{\alpha} d_{1} t_{1}^{2}}{8}+ \\
& -2 \Gamma \lambda t_{1}^{3} t_{2}+\frac{\Gamma \hat{\alpha} t_{2}^{2} t_{3}}{16}+\frac{\Gamma \hat{\alpha} d_{2} t_{1}^{2}}{4}+\frac{\Gamma \hat{\alpha} t_{1} t_{2} t_{4}}{4}, \\
& t_{17}=-\frac{\Gamma t_{1} t_{9}}{2}+\frac{\Gamma \hat{\alpha} t_{1}^{2} t_{5}}{4}, \quad t_{18}=-\frac{\Gamma t_{1} t_{10}}{2}-\frac{\Gamma \hat{\alpha} t_{1}^{2} t_{5}}{4}+\frac{\Gamma \hat{\alpha} d_{1} t_{1}^{2}}{4}, \\
& t_{19}=-\Gamma t_{2} t_{8}-\Gamma t_{1} e_{1}-\frac{9 \Gamma t_{1} t_{11}}{2}+\frac{\Gamma t_{10} t_{2}}{2}-\frac{\Gamma t_{9} t_{2}}{2}+2 \Gamma t_{1} t_{12}+ \\
& +\Gamma \hat{\alpha} d_{2} t_{1} t_{2}-\Gamma \hat{\alpha} d_{1} t_{1} t_{2}+\frac{3 \Gamma \hat{\alpha} t_{1} t_{2} t_{5}}{2}+\frac{\Gamma \hat{\alpha} t_{2}^{2} t_{4}}{4}-6 \Gamma \lambda t_{1}^{2} t_{2}^{3}, \\
& t_{20}=-\Gamma t_{2} t_{9}-3 \Gamma t_{1} t_{4}+\Gamma \hat{\alpha} t_{1} t_{2} t_{5}, \\
& t_{21}=-\Gamma t_{2} t_{9}-\Gamma t_{10} t_{2}-2 \Gamma t_{1} t_{12}+6 \Gamma t_{1} t_{11}+\Gamma \hat{\alpha} d_{1} t_{1} t_{2}-2 \Gamma \hat{\alpha} t_{1} t_{2} t_{5}, \\
& t_{22}=\frac{\Gamma \hat{\alpha} t_{2}^{2} t_{5}}{12}-\frac{\Gamma t_{11} t_{2}}{12} \\
& t_{23}=\frac{\Gamma \hat{\alpha} d_{1} t_{2}^{2}}{6}-\frac{2 \Gamma t_{12} t_{2}}{3}, \quad t_{24}=\frac{\Gamma \hat{\alpha} d_{2} t_{2}^{2}}{2}-4 \Gamma t_{1} t_{2}^{3}+4 \Gamma t_{1} t_{13}-\Gamma e_{1} t_{2}, \\
& t_{25}=-\frac{\Gamma \lambda t_{2}^{4}}{4}+\Gamma t_{2} t_{13} \\
& d_{1}=-\frac{t_{3} R^{4}+t_{4} R^{2}+t_{5} \ln (R)^{2}-t_{3}-t_{4}}{\ln (R)}, \quad d_{2}=-\left(t_{3}+t_{4}\right), \\
& e_{1}=\frac{-t_{11} R^{2} \ln (R)^{3}-\ln (R)^{2}\left(t_{9} R^{4}+t_{12} R^{2}\right)-t_{10} R^{4} \ln (R)}{R^{2} \ln (R)}+ \\
& -\frac{\left(t_{6} R^{6}+R^{2}\left(t_{4}+t_{7}\right)+R^{4}\left(t_{6}+t_{7}+t_{8}\right)-t_{13}\right)(R+1)(R-1)}{R^{2} \ln (R)},
\end{aligned}
$$




$$
\begin{aligned}
& e_{2}=-\left(t_{6}+t_{7}+t_{8}+t_{13}\right), \\
& f_{1}=-\frac{t_{14} R^{8}}{\ln (R)}-\frac{t_{15} R^{6}}{\ln (R)}-\frac{R^{4}\left(t_{16}+t_{18} \ln (R)+t_{17} \ln (R)^{2}\right)}{\ln (R)}-\frac{R^{2}\left(t_{19}+t_{21} \ln (R)+t_{20} \ln (R)^{2}\right)}{\ln (R)}+ \\
& -\frac{t_{25}}{R^{2} \ln (R)}-\frac{t_{22} \ln (R)^{4}+t_{23} \ln (R)^{3}+t_{24} \ln (R)^{2}-t_{14}-t_{15}-t_{16}-t_{19}-t_{25}}{\ln (R)}, \\
& f_{2}=-\left(t_{14}+t_{15}+t_{16}+t_{19}+t_{25}\right) .
\end{aligned}
$$

For $\hat{\alpha}=0$, solutions are reduced to those of constant viscosity case.

Case 2: For space dependent viscosity [11], here

$$
\mu=r,
$$

we shall select

$$
\Lambda=\varepsilon \lambda, \quad M=\varepsilon m .
$$

Due to the nonlinear nature of the velocity and temperature field Eqs in (2.10)-(2.12) and, it is convenient to form a power series expansion both in parameter $\varepsilon$,

$$
\begin{aligned}
& u_{z}=u_{z}^{0}+\varepsilon u_{z}^{1}+O\left(\varepsilon^{2}\right), \\
& \theta=\theta_{0}+\varepsilon \theta_{1}+O\left(\varepsilon^{2}\right)
\end{aligned}
$$

Substituting these forms of expansion and equating coefficients of these power terms, one obtains the following terms:

For $O(1)$ :

$$
\begin{aligned}
& r\left(u_{z}^{0}\right)^{\prime \prime}+2\left(u_{z}^{0}\right)^{\prime}=C, \\
& \theta_{0}^{\prime \prime}+\frac{1}{r} \theta_{0}^{\prime}=-\Gamma r\left(\left(u_{z}^{0}\right)^{\prime}\right)^{2}, \\
& u_{z}^{0}(1)=0, \quad \theta(1)=0 .
\end{aligned}
$$

For $O(\varepsilon)$ :

$$
\begin{aligned}
& r\left(u_{z}^{l}\right)^{\prime \prime}+2\left(u_{z}^{l}\right)^{\prime}=\frac{-\lambda\left(\left(u_{z}^{0}\right)^{\prime}\right)^{3}}{r}-3 \lambda\left(\left(u_{z}^{0}\right)^{\prime}\right)^{2}\left(u_{z}^{0}\right)^{\prime \prime}+m u_{z}^{0}, \\
& \theta_{1}^{\prime \prime}+\frac{1}{r} \theta_{1}^{\prime}=-\Gamma \lambda\left(\left(u_{z}^{0}\right)^{\prime}\right)^{4}-2 \Gamma r\left(u_{z}^{0}\right)^{\prime}\left(u_{z}^{l}\right)^{\prime}, \\
& u_{z}^{l}(R)=0, \quad \theta_{l}(R)=0 .
\end{aligned}
$$


The following results are obtained when Eq.(3.26) is solved first and then Eq.(3.27) is solved.

$$
\begin{aligned}
& u_{z}^{0}=t_{1}\left(\frac{R}{r}+r\right)+t_{2}, \\
& \theta_{0}=t_{3} r^{3}+t_{4} r+\frac{t_{5}}{r}+d_{1} \ln (r)+d_{2}, \\
& u_{z}^{1}=t_{6} r^{2}+\frac{t_{7}}{r^{4}}+t_{8} \ln (r)+t_{9} r+\frac{t_{10}}{r^{2}}+\frac{t_{11}}{r^{6}}-\frac{e_{1}}{r}+e_{2}, \\
& \theta_{1}=t_{12} r^{4}+t_{13} r^{3}+t_{14} r^{2}+t_{15} r+\frac{t_{16}}{r^{6}}+\frac{t_{17}}{r^{4}}+\frac{t_{18}}{r^{2}}+\frac{t_{19}}{r}+t_{20} \ln (r)^{2}+f_{1} \ln (r)+f_{2}
\end{aligned}
$$

where $t_{1,2,3 \ldots 20}, d_{1}, d_{2}, e_{1}, e_{2}, f_{1}$ and $f_{2}$ are

$$
\begin{aligned}
& t_{1}=\frac{C}{2}, t_{2}=-\frac{C}{2}(R+1), t_{3}=-\frac{\Gamma t_{1}^{2}}{9}, t_{4}=2 \Gamma R t_{1}^{2}, t_{5}=-\Gamma R^{2} t_{1}^{2}, t_{6}=\frac{m t_{1}}{6}, t_{7}=\frac{3 t_{1}^{3} R^{2} \lambda}{4}, \\
& t_{8}=m R t_{1}-\lambda t_{1}^{3}, t_{9}=\frac{m t_{2}}{2}, t_{10}=-\frac{3 \lambda R t_{1}^{3}}{4}, t_{11}=-\frac{\lambda R^{3} t_{1}^{3}}{6}, t_{12}=-\frac{\Gamma t_{1} t_{6}}{4}, t_{13}=-\frac{2 \Gamma t_{1} t_{9}}{9} \\
& t_{14}=-\frac{\Gamma \lambda t_{1}^{4}}{4}+\Gamma R t_{1} t_{6}-\frac{\Gamma t_{1} t_{8}}{2}, \quad t_{15}=2 \Gamma R t_{1} t_{9}-2 \Gamma e_{1} t_{1}, t_{16}=-\frac{\Gamma \lambda R^{4} t_{1}^{4}}{36}-\frac{\Gamma R t_{1} t_{11}}{3}, \\
& t_{17}=\frac{\Gamma \lambda R^{3} t_{1}^{4}}{4}+\frac{3 \Gamma t_{1} t_{11}}{4}-\frac{\Gamma R^{3} t_{1} t_{7}}{2}, t_{18}=-\frac{3 \Gamma \lambda R^{2} t_{1}^{4}}{2}-\Gamma R t_{1} t_{10}+2 \Gamma t_{1} t_{7}, t_{19}=2 e_{1} \Gamma R t_{1} \\
& t_{20}=2 \Gamma \lambda R t_{1}^{4}+2 \Gamma t_{1} t_{10}+\Gamma R t_{1} t_{8}, \quad d_{1}=\frac{(R-1)\left(t_{3} R^{3}+t_{3} R^{2}+R\left(t_{3}+t_{4}\right)-t_{5}\right)}{R \ln (R)} \\
& d_{2}=-\left(t_{3}+t_{4}+t_{5}\right), \\
& e_{1}=\frac{-t_{8} R^{6} \ln (R)-t_{6} R^{8}-t_{9} R^{7}+R^{6}\left(t_{6}+t_{7}+t_{9}+t_{10}+t_{11}\right)-t_{10} R^{4}-t_{7} R^{2}-t_{11}}{R^{5}(R-1)} \\
& e_{2}=\frac{-t_{8} R^{6} \ln (R)-t_{6} R^{8}-t_{9} R^{7}+R^{5}\left(t_{6}+t_{7}+t_{9}+t_{10}+t_{11}\right)-t_{10} R^{4}-t_{7} R^{2}-t_{11}}{R^{5}(R-1)}
\end{aligned}
$$




$$
\begin{gathered}
f_{1}=-\frac{t_{12} R^{4}}{\ln (R)}-\frac{t_{13} R^{3}}{\ln (R)}-\frac{t_{14} R^{2}}{\ln (R)}-\frac{t_{15} R}{\ln (R)}+\frac{-t_{20} \ln (R)^{2}+t_{12}+t_{13}+t_{14}+t_{15}+t_{16}+t_{17}+t_{18}+t_{19}}{\ln (R)}+ \\
-\frac{t_{19}}{R \ln (R)}-\frac{t_{18}}{R^{2} \ln (R)}-\frac{t_{17}}{R^{4} \ln (R)}-\frac{t_{16}}{R^{6} \ln (R)}, \\
f_{2}=-\left(t_{12}+t_{13}+t_{14}+t_{15}+t_{16}+t_{17}+t_{18}+t_{19}\right) .
\end{gathered}
$$

In the next section, the perturbation solutions in these two cases will be contrasted with those generated numerically by the software package Matlab.

\section{Numerical results}

The non-dimensional forms of the coupled Eqs (2.10) and (2.11) with the conditions (2.12) are solved numerically for the Reynolds viscosity model and space dependent viscosity. In Figs 2 and 3, numerical and the new analytical solutions are compared and an excellent agreement is observed. The comparison of the results was made for both the velocity profile and temperature profile. Figures $4 a$ and $b$ show the effect of the MHD parameter on the fluid flow. In Fig.4a, it is clear that the effects on the temperature profile are such that the temperature decreases as the parameter increases. Figure $4 \mathrm{~b}$ shows that the effect of the MHD parameter on the velocity profile is similar. In Figs 5a and b, we can see the effects of temperature and velocity on the non-Newtonian parameter. When $\Lambda=0$, the fluid shows a Newtonian behavior. As can be seen from both figures, as the value of non-Newtonian character increases, temperature and velocity of the fluid decrease. Another important result from the figures is that the rate of fluid strain in the pipe walls is higher because the non-Newtonian parameter is inversely proportional to the viscosity of the fluid. Figures $6 \mathrm{a}$ and $6 \mathrm{~b}$ suggest other important results. For example, Fig.6a shows the effect of the viscosity parameter on the temperature profile. It is seen that the temperature increases in the fluid with the increase of the parameter for the Reynolds viscosity model. In the Reynolds viscosity model, the viscosity decreases as the temperature increases since $\alpha=0$ corresponds to constant viscosity state. In Fig.6b, it is seen that velocity increases with an increment in the viscosity parameter. The effects of non-Newtonian parameters on the temperature profile and velocity profile for the space dependent viscosity model are displayed in Figs 7a and $b$, respectively.

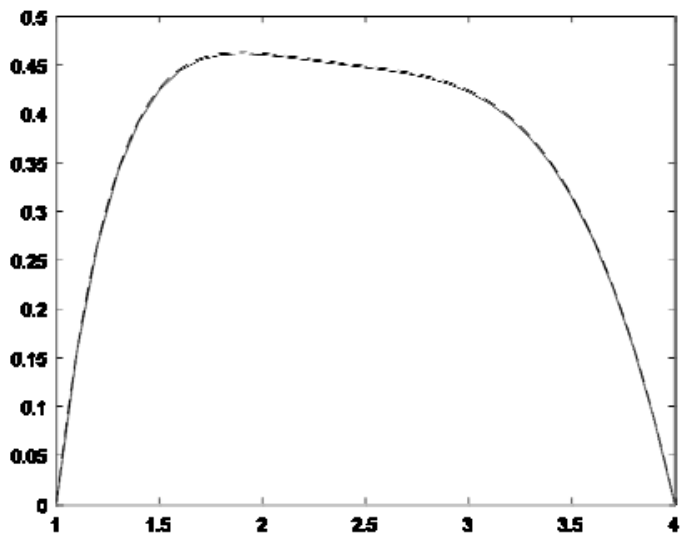

(a)

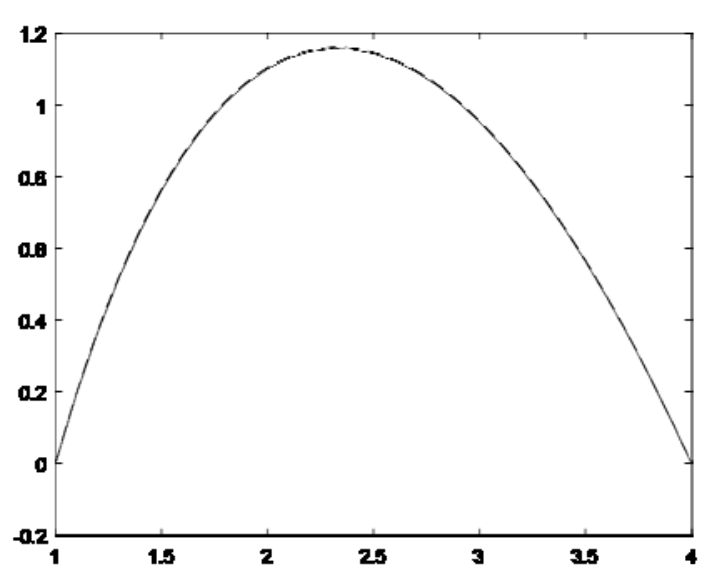

(b)

Fig.2ab. Comparison of the perturbation solution with the numerical solution for the case of the Reynolds viscosity model. (------- Numerical solution , Analytical solution) (a) Temperature profile, (b) Velocity profile, $(C=-1 ; \Gamma=1 ; \Lambda=0.01 ; M=0.01 ; \alpha=0.01)$. 


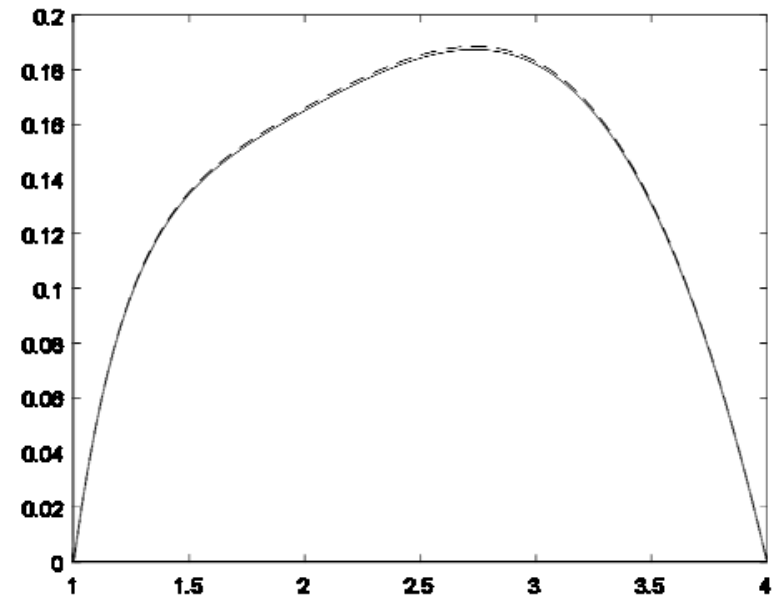

(a)

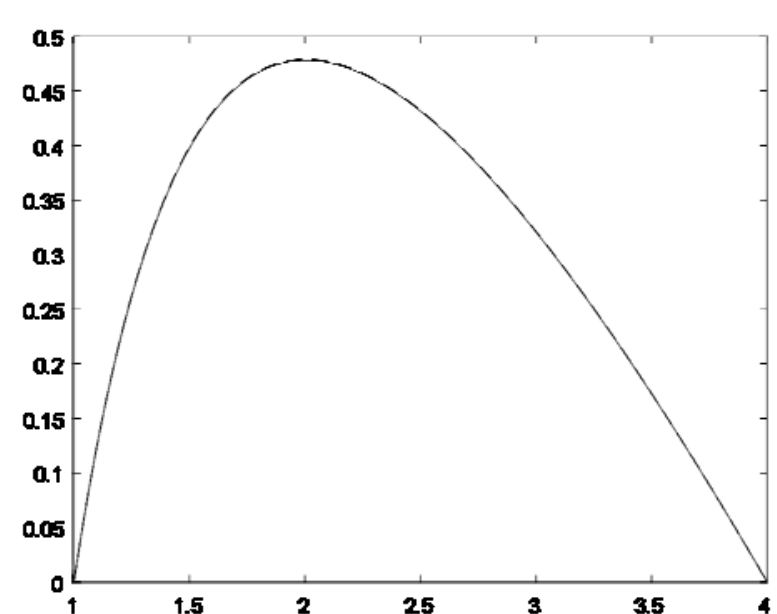

(b)

Fig.3ab. Comparison of the perturbation solution with the numerical solution for the case of the space dependent viscosity model. (------ Numerical solution,

Analytical solution)

(a) Temperature profile, (b) Velocity profile, $(C=-1 ; \Gamma=1 ; \Lambda=0.01 ; M=0.1)$.

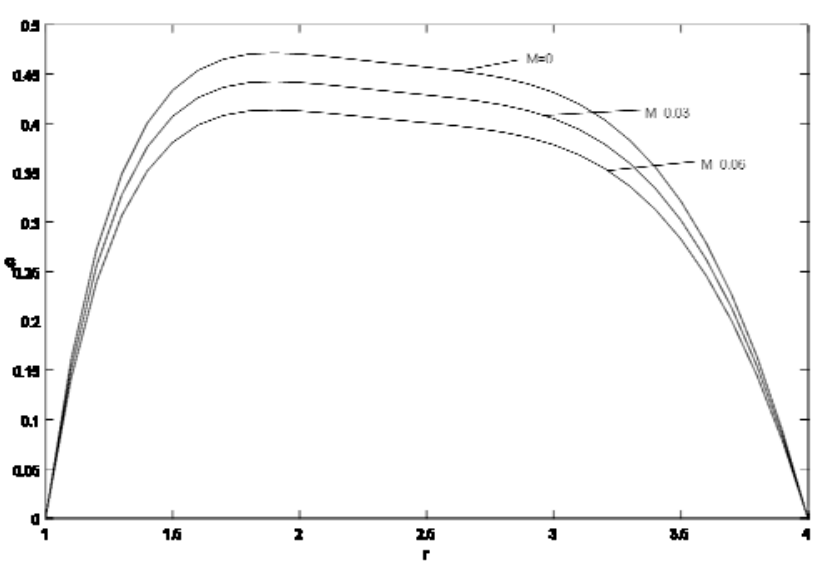

(a)

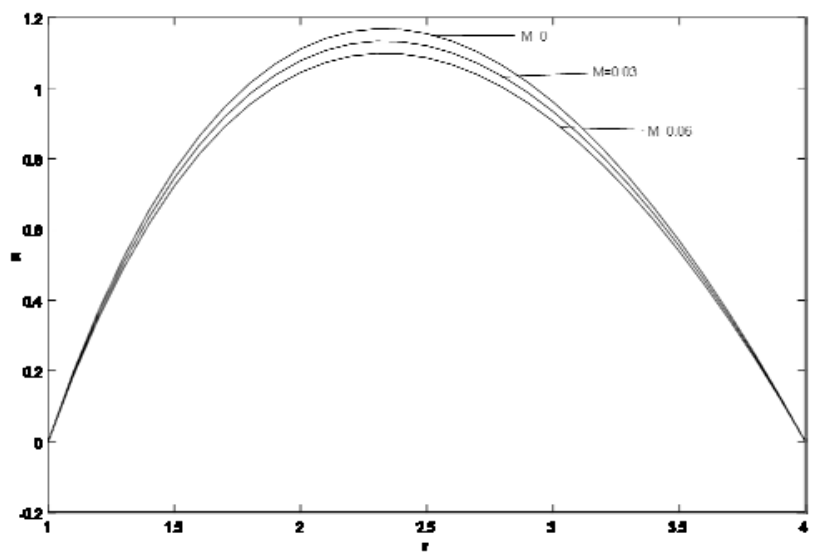

(b)

Fig.4ab. Influence of the MHD parameter for the Reynolds viscosity model on (a) Temperature profile, (b) Velocity profile, $(C=-1 ; \Gamma=1 ; \Lambda=0.01 ; \alpha=0.01)$.

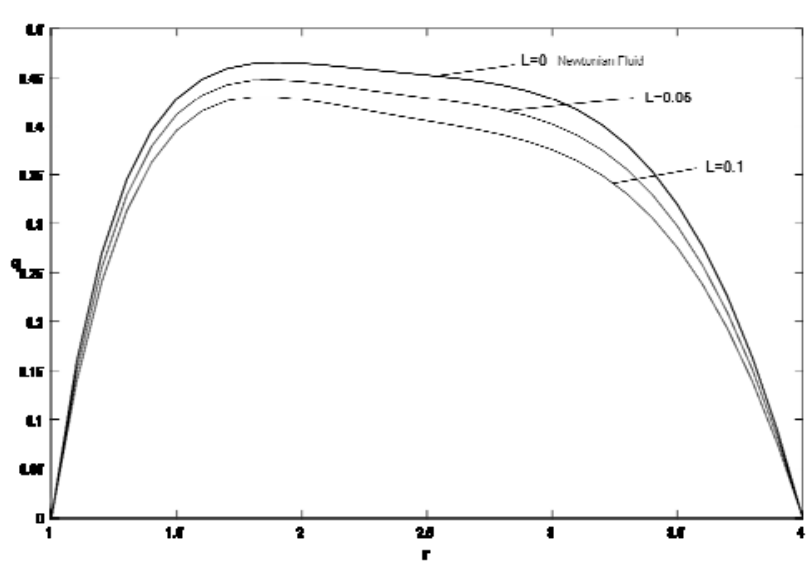

(a)

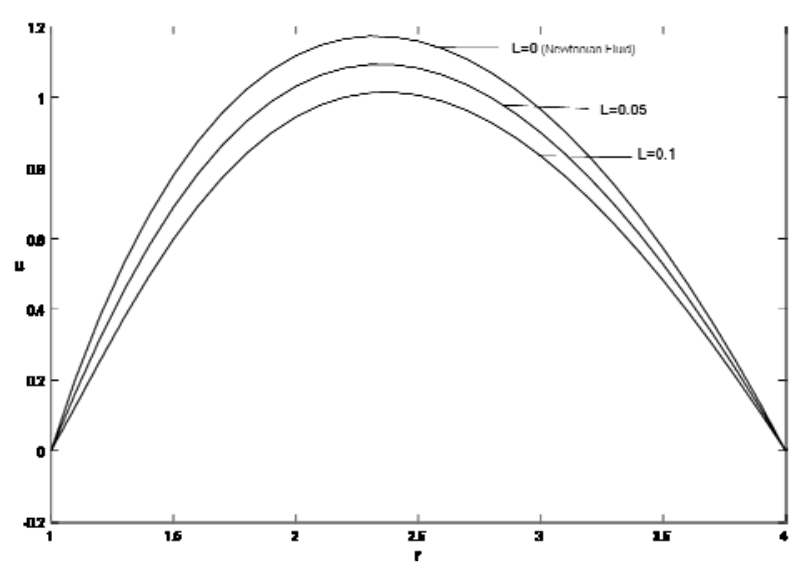

(b)

Fig.5ab. Influence of the non-Newtonian parameter for the Reynolds viscosity model on (a) Temperature profile, (b) Velocity profile, $(C=-1 ; \Gamma=1 ; M=0.01 ; \alpha=0.01)$. 


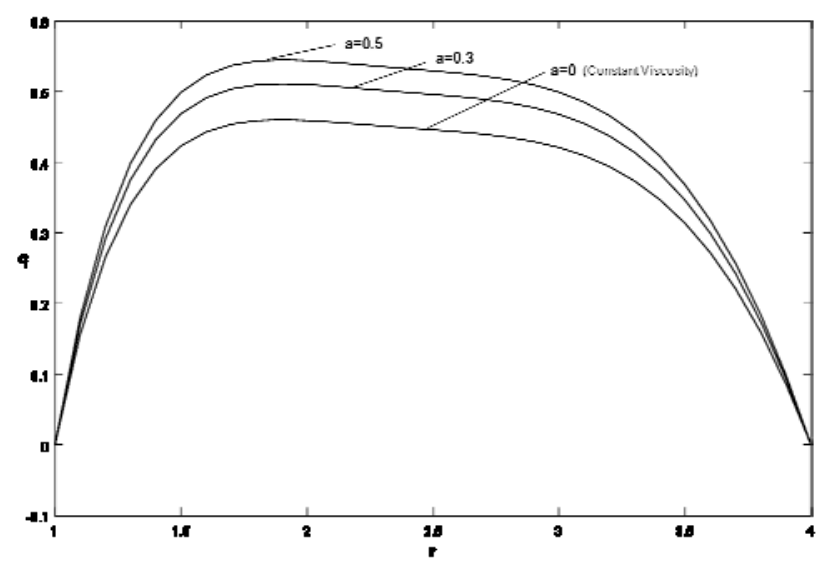

(a)

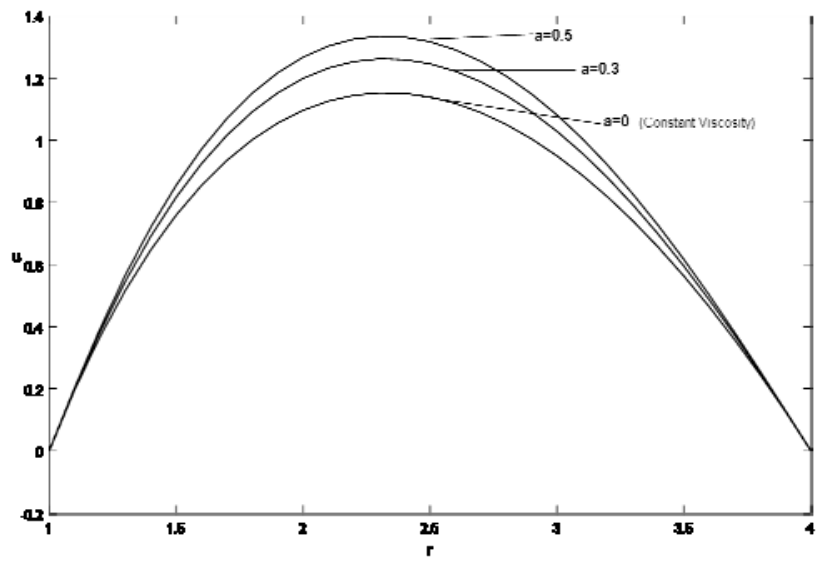

(b)

Fig.6ab. Influence of the viscosity parameter for the Reynolds viscosity model on (a) Temperature profile, (b) Velocity profile, $(C=-1 ; \Gamma=1 ; M=0.01 ; \Lambda=0.01)$.

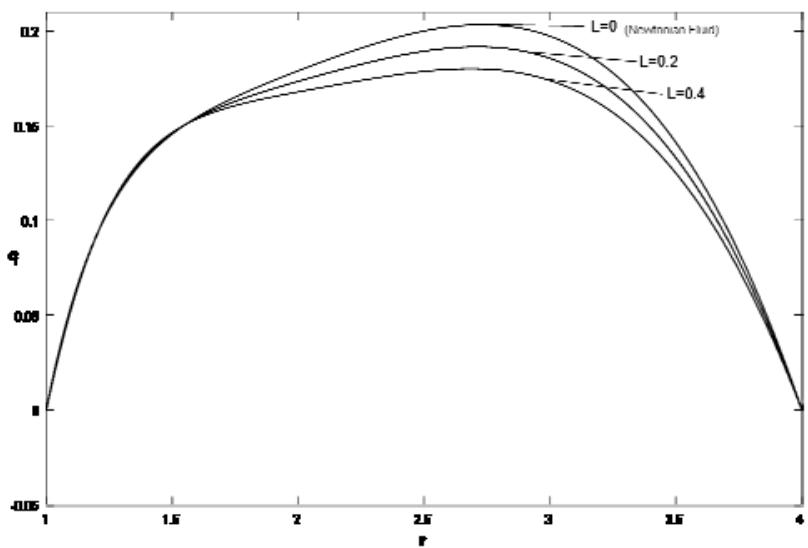

(a)

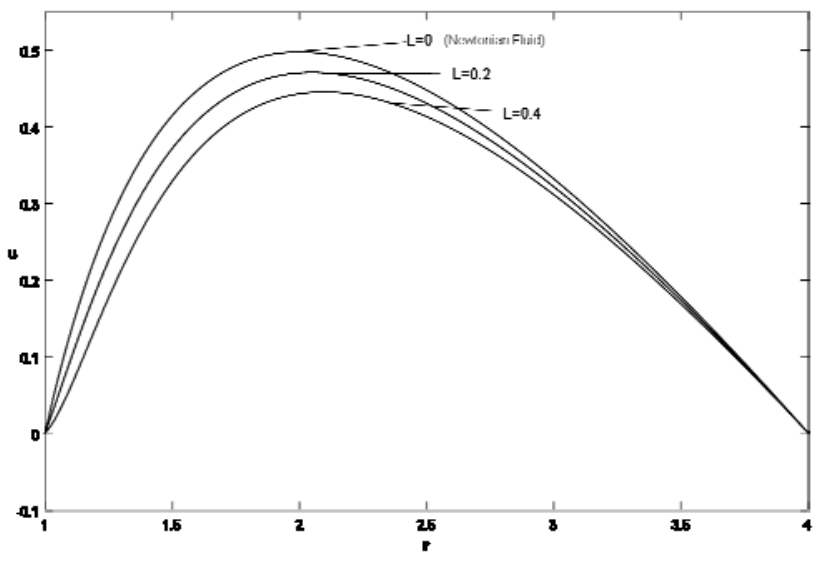

(b)

Fig.7ab. Influence of the non-Newtonian parameter for the space dependent viscosity model on (a) Temperature profile, (b) Velocity profile, $(C=-1 ; \Gamma=1 ; M=0.01)$.

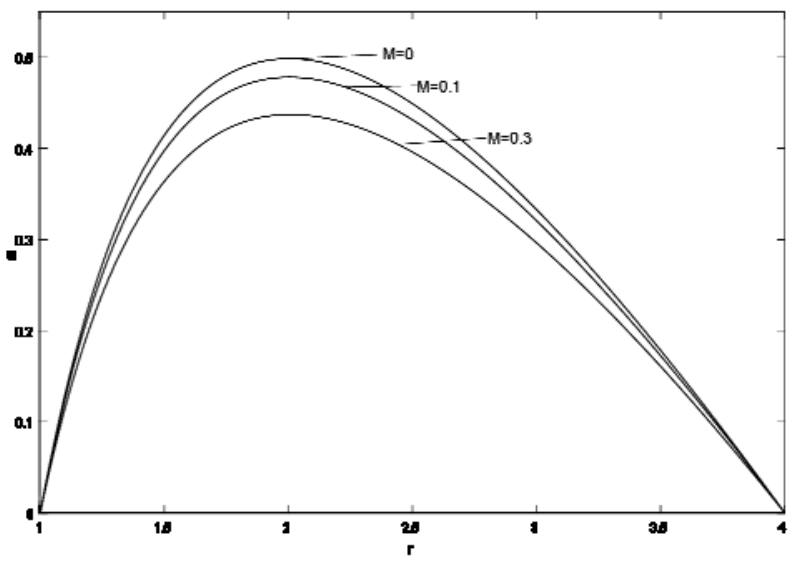

(a)

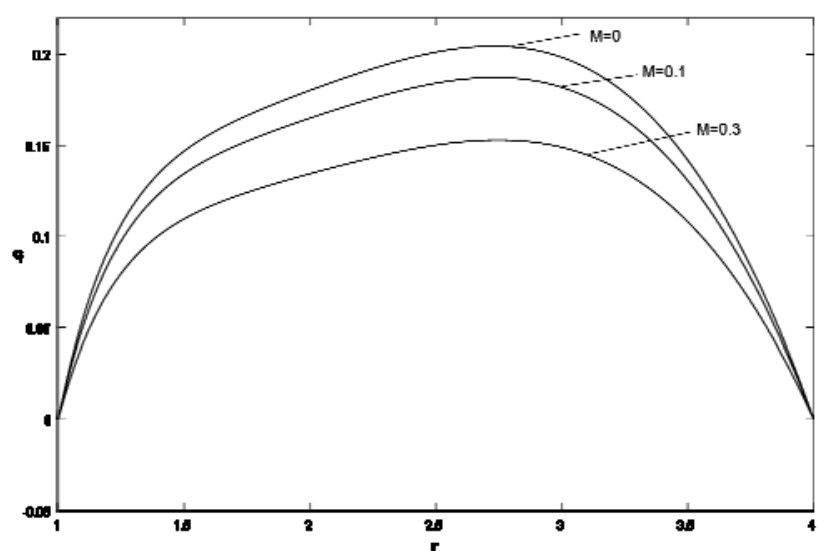

(b)

Fig.8ab. Influence of the MHD parameter for the space dependent viscosity model on (a) Temperature profile, (b) Velocity profile, $(C=-1 ; \Gamma=1 ; \Lambda=0.01)$. 
Similarly, effects of the MHD parameter are shown in Fig.8. Figure 7a shows that as the value of $\Lambda$ increases, the temperature of the fluid decreases. Unlike the Reynolds model, the maximum temperature was observed near the outer pipe. Figure $7 \mathrm{~b}$ shows the effect of $\Lambda$ on the velocity profile, the parameter increases as velocity decreases. Figures $8 \mathrm{a}$ and $\mathrm{b}$ display the temperature and velocity profiles, respectively, while the variable parameter is the MHD parameter. As can be seen from both Figs 7 and 8, as the MHD parameter increases, the temperature and velocity decrease.

\section{Conclusions}

In this paper, we have investigated the influence of MHD on the Reynolds viscosity and space viscosity models in a third-grade fluid behavior in an annular pipe. Approximate analytical solutions have been generated for both viscosity models using the perturbation method. In the solutions, the variation of velocity and temperature profiles for MHD and non-Newtonian fluid parameters are also presented. In addition, analytical solutions are compared with numerical solutions and an excellent agreement is observed. In both viscosity models, both non-Newtonian parameters increase and velocity and temperature have been observed to be decreasing in the fluid. In both viscosity models, the increase in the non-Newtonian parameter has been observed to reduce the magnitude of velocity and temperature. However, a similar influence was obtained with a decrease in the viscosity parameter for the Reynolds model. When the effects of the MHD parameter on the velocity and temperature profiles are investigated, the magnitude of temperature and velocity are decreased as the MHD parameter is increased in both viscosity models. In all figures (Figs 3b$8 \mathrm{~b})$ containing velocity curves, it is demonstrated that the maximum velocity reaches close to the inner pipe. In all Figures (Figs 3a-8a) containing temperature curves, it is demonstrated that the maximum temperature in the Reynolds viscosity model is obtained close to the inner pipe, whereas in the other viscosity model it is observed near the outer pipe.

\section{Nomenclature}

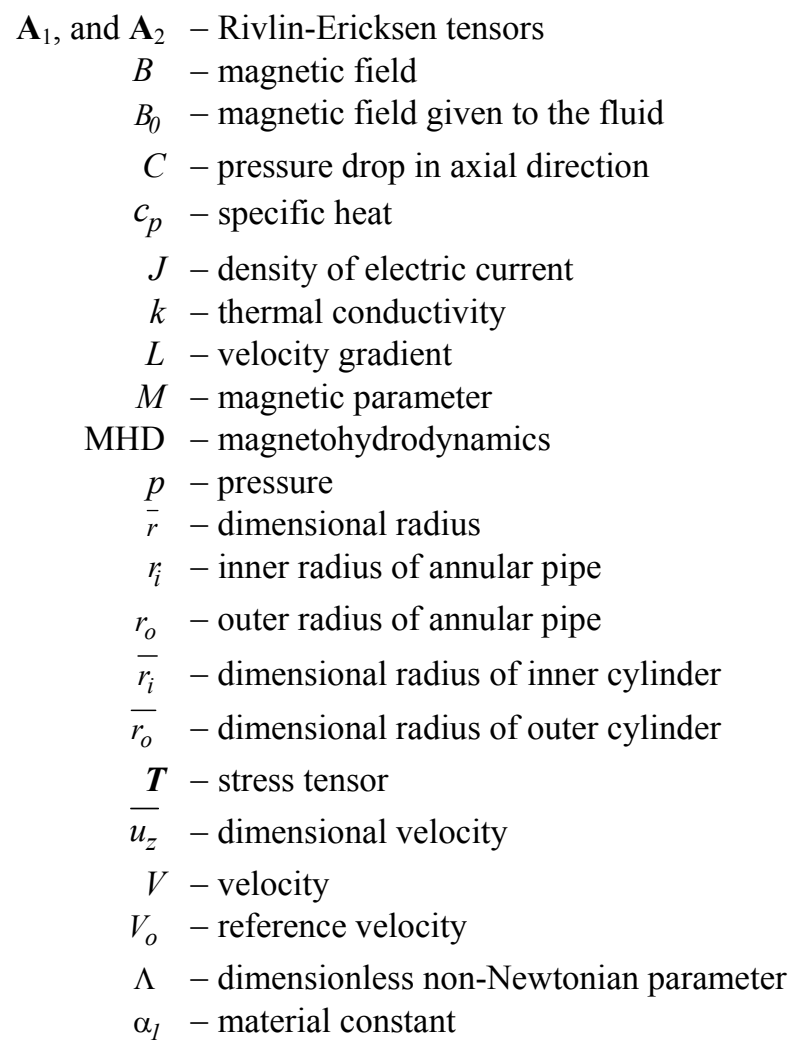




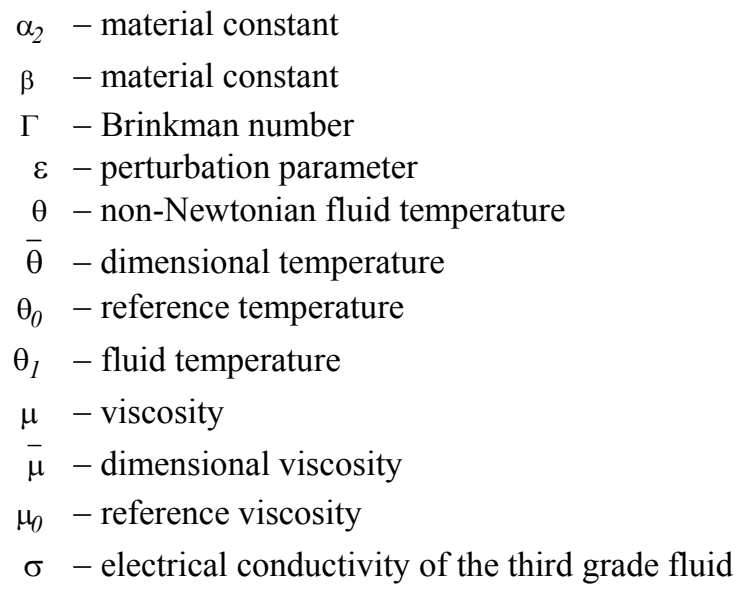

\section{References}

[1] Yürüsoy M. and Pakdemirli M. (1999): Exact solutions of boundary layer equations of a special non-Newtonian fluid over a stretching sheet. - Mechanics Research Communications, vol.26, No.2, pp.171-175.

[2] Pakdemirli M. (1994): Conventional and multiple deck boundary layer approach to second and third grade fluids. - Int. J. Engng Sci., vol.32, 141.

[3] Hayat T. and Kara A.H. (2006): Couette flow of a third-grade fluid with variable magnetic field. - Mathematical and Computer Modelling, vol.43, pp.132-137.

[4] Hayat T., Shahzad F. and Ayub M. (2007): Analytical solution for the steady flow of the third grade fluid in a porous half space. - Applied Mathematical Modelling, vol.31, pp.2424-2432.

[5] Pakdemirli M., Hayat T., Yürüsoy M., Abbasbandy S. and Asghar S. (2011): Perturbation analysis of a modified second grade fluid over a porous plate. - Nonlinear Analysis: Real World Applications, vol.12, pp.1774-785.

[6] Pakdemirli M., Aksoy Y., Yürüsoy M. and Khalique C.M. (2008): Symmetries of boundary layer equations of power-law fluids of second grade. - Acta Mech Sin., vol.24, pp.661-670.

[7] Ali J. Chamkha (1997): Similarity solution for thermal boundary layer on a stretched surface of a non-Newtonian fluid. - Int. Comm. Heat Mass Transfer, vol.24, No.5, pp.643-652.

[8] Kecebas A. and Yürüsoy M. (2006): Similarity solutions of unsteady boundary layer equations of a special third grade fluid. - International Journal of Engineering Science, vol.44, pp.721-729.

[9] Massoudi M. and Christie I. (1995): Effects of variable viscosity and viscous dissipation on the flow of a thirdgrade fluid in a pipe. - Int. J. Non-Linear Mech., vol.30, pp.687-699.

[10] Yürüsoy M. and Pakdemirli M. (2002): Approximate analytical solutions for the flow of a third-grade fluid in a pipe. - International Journal of Non-Linear Mechanics, vol.37, pp.187-195.

[11] Ellahi R. and Riaz A. (2010): Analytical solutions for MHD flow in a third-grade fluid with variable viscosity. Mathematical and Computer Modelling, vol.52, pp.1783-1793.

[12] Akinshilo A.T. and Olaye O.: On the analysis of the Erying Powell model based fluid flow in a pipe with temperature dependent viscosity and internal heat generation. - Journal of King Saud University - Engineering Sciences, (to be published).

[13] Jayeoba O.J. and Okoya S.S. (2012): Approximate analytical solutions for pipe flow of a third grade fluid with variable models of viscosities and heat generation/ absorption. - Journal of the Nigerian Mathematical Society, vol.31, pp.207-227. 